\title{
On the behavior of WOWA operators
}

\author{
Bonifacio Llamazares \\ University of Valladolid, PRESAD Research Group, 47011 Valladolid, Spain, \\ boni@eco.uva.es, http://www2.eco.uva.es/boni
}

\begin{abstract}
In this paper we analyze the behavior of WOWA operators, a class of functions that simultaneously generalize weighted means and OWA operators. Moreover, we introduce functions that also generalize both operators and characterize those satisfying a condition imposed to maintain the relationship among the weights.
\end{abstract}

Keywords: WOWA operators, OWA operators, weighted means.

\section{Introduction}

Weighted means and ordered weighted averaging (OWA) operators (Yager [8]) are aggregation functions widely used in the literature. Weighted means allow to weight each information source in relation to their reliability while OWA operators allow to weight the values according to their ordering. The need to combine both functions has been reported by several authors (see, among others, Torra [4] and Torra and Narukawa [6]). In [4], Torra introduces the weighted OWA (WOWA) operator, a new aggregation function that allows to combine both weights.

The aim of this paper is to analyze the behavior of WOWA operators. Moreover, since, in some cases, the results provided by these operators may be questionable, we propose to use functions that maintain the relationship among the weights of a weighting vector when the non-zero components of the other weighting vector are equal. In this way, we obtain a class of functions that have been previously introduced by Engemann et al. [1] in a different framework.

The paper is organized as follows. In Section 2 we introduce weighted means, OWA operators and WOWA operators. Section 3 shows some questionable behaviors of WOWA operators. In Section 4 we propose a condition to maintain the relationship among the weights and characterize the functions that satisfy this condition. The paper concludes in Section 5 .

\section{Preliminaries}

Throughout the paper we will use the following notation: vectors will be denoted in bold; $\boldsymbol{\eta}$ will denote the vector $(1 / n, \ldots, 1 / n) ; \boldsymbol{x} \geq \boldsymbol{y}$ will mean $x_{i} \geq y_{i}$ for all $i \in\{1, \ldots, n\}$; given $\sigma$ a permutation of $\{1, \ldots, n\}, \boldsymbol{x}_{\sigma}$ will denote the vector $\left(x_{\sigma(1)}, \ldots, x_{\sigma(n)}\right)$. 
In the following definition we present some well-known properties usually demanded to the functions used in the aggregation processes.

Definition 1. Let $F: \mathbb{R}^{n} \longrightarrow \mathbb{R}$ be a function.

1. $F$ is symmetric if $F\left(\boldsymbol{x}_{\sigma}\right)=F(\boldsymbol{x})$ for all $\boldsymbol{x} \in \mathbb{R}^{n}$ and for all permutation $\sigma$ of $\{1, \ldots, n\}$.

2. $F$ is monotonic if $F(\boldsymbol{x}) \geq F(\boldsymbol{y})$ for all $\boldsymbol{x}, \boldsymbol{y} \in \mathbb{R}^{n}$ such that $\boldsymbol{x} \geq \boldsymbol{y}$.

3. $F$ is idempotent if $F(x, \ldots, x)=x$ for all $x \in \mathbb{R}$.

4. $F$ is compensative if $\min (\boldsymbol{x}) \leq F(\boldsymbol{x}) \leq \max (\boldsymbol{x})$ for all $\boldsymbol{x} \in \mathbb{R}^{n}$.

5. $F$ is homogeneous of degree 1 if $F(\lambda \boldsymbol{x})=\lambda F(\boldsymbol{x})$ for all $\boldsymbol{x} \in \mathbb{R}^{n}$ and for all $\lambda>0$.

\subsection{Weighted means and OWA operators}

Weighted means and OWA operators are defined by vectors with non-negative components whose sum is 1 .

Definition 2. A vector $\boldsymbol{\mu} \in \mathbb{R}^{n}$ is a weighting vector if $\boldsymbol{\mu} \in[0,1]^{n}$ and $\sum_{i=1}^{n} \mu_{i}=1$.

Definition 3. Let $\boldsymbol{p}$ be a weighting vector. The weighted mean associated with $\boldsymbol{p}$ is the function $F_{\boldsymbol{p}}: \mathbb{R}^{n} \longrightarrow \mathbb{R}$ given by

$$
F_{\boldsymbol{p}}\left(x_{1}, \ldots, x_{n}\right)=\sum_{i=1}^{n} p_{i} x_{i} .
$$

The weighted means are monotonic, idempotent, compensative and homogeneous of degree 1 functions.

In [8], Yager introduced OWA operators as a tool for aggregation procedures in multicriteria decision making.

Definition 4. Let $\boldsymbol{w}$ be a weighting vector. The OWA operator associated with $\boldsymbol{w}$ is the function $F^{\boldsymbol{w}}: \mathbb{R}^{n} \longrightarrow \mathbb{R}$ given by

$$
F^{\boldsymbol{w}}\left(x_{1}, \ldots, x_{n}\right)=\sum_{i=1}^{n} w_{i} x_{\sigma(i)},
$$

where $\sigma$ is a permutation of $\{1, \ldots, n\}$ such that $x_{\sigma(1)} \geq \cdots \geq x_{\sigma(n)}$.

OWA operators are symmetric, monotonic, idempotent, compensative and homogeneous of degree 1 functions.

One of the most important issues in the theory of OWA operators is the determination of associated weights (see, for instance, Xu [7] and Fullér [2]). In [9], Yager relates the OWA operators weights to quantifiers.

Definition 5. A quantifier is a non-decreasing function $Q:[0,1] \longrightarrow[0,1]$ that satisfies $Q(0)=0$ and $Q(1)=1$. 
Given a quantifier $Q$, the OWA operator weights can be obtained from the expression $w_{i}=Q(i / n)-Q((i-1) / n), \quad i=1, \ldots, n$, (Yager [9]). From this relation follows:

$$
Q\left(\frac{i}{n}\right)=\sum_{j=1}^{i} w_{j}, \quad i=1, \ldots, n
$$

i.e., the same weighting vector can be obtained through any quantifier interpolating the points $\left(i / n, \sum_{j=1}^{i} w_{j}\right), i=1, \ldots, n$.

\subsection{WOWA operators}

WOWA operators were introduced by Torra [4] in order to consider situations where both the importance of information sources and the importance of values had to be taken into account.

Definition 6. Let $\boldsymbol{p}$ and $\boldsymbol{w}$ be two weighting vectors. The WOWA operator associated with $\boldsymbol{p}$ and $\boldsymbol{w}$ is the function $W_{\boldsymbol{p}}^{\boldsymbol{w}}: \mathbb{R}^{n} \longrightarrow \mathbb{R}$ given by

$$
W_{\boldsymbol{p}}^{\boldsymbol{w}}\left(x_{1}, \ldots, x_{n}\right)=\sum_{i=1}^{n} \mu_{i} x_{\sigma(i)},
$$

where $\sigma$ is a permutation of $\{1, \ldots, n\}$ such that $x_{\sigma(1)} \geq \cdots \geq x_{\sigma(n)}$ and the weight $\mu_{i}$ is defined as

$$
\mu_{i}=f\left(\sum_{j=1}^{i} p_{\sigma(j)}\right)-f\left(\sum_{j=1}^{i-1} p_{\sigma(j)}\right),
$$

where $f$ is a non-decreasing function that interpolates the points $\left(i / n, \sum_{j=1}^{i} w_{j}\right)$ together with the point $(0,0)$. Moreover, $f$ is the identity when the points can be interpolated in this way.

Any quantifier generating the weighting vector $\boldsymbol{w}$ satisfies the required properties of the function $f$ in the previous definition (under the assumption that the quantifier is the identity when $\boldsymbol{w}=\boldsymbol{\eta}$ ). For this reason, it is possible to give an alternative definition of WOWA operators using quantifiers (Torra and Godo [5]).

Definition 7. Let $\boldsymbol{p}$ be a weighting vector and let $Q$ be a quantifier. The WOWA operator associated with $\boldsymbol{p}$ and $Q$ is the function $W_{\boldsymbol{p}}^{Q}: \mathbb{R}^{n} \longrightarrow \mathbb{R}$ given by

$$
W_{\boldsymbol{p}}^{Q}\left(x_{1}, \ldots, x_{n}\right)=\sum_{i=1}^{n} \mu_{i} x_{\sigma(i)},
$$


where $\sigma$ is a permutation of $\{1, \ldots, n\}$ such that $x_{\sigma(1)} \geq \cdots \geq x_{\sigma(n)}$ and the weight $\mu_{i}$ is defined as

$$
\mu_{i}=Q\left(\sum_{j=1}^{i} p_{\sigma(j)}\right)-Q\left(\sum_{j=1}^{i-1} p_{\sigma(j)}\right) .
$$

WOWA operators are monotonic, idempotent, compensative and homogeneous of degree 1 functions. Moreover, $W_{\boldsymbol{p}}^{\boldsymbol{\eta}}=F_{\boldsymbol{p}}$ and $W_{\boldsymbol{\eta}}^{\boldsymbol{w}}=F^{\boldsymbol{w}}$ (Torra [4]).

\section{Analysis of WOWA operators}

In this section we show with examples some questionable behaviors of WOWA operators.

Example 1. Suppose we have five sensors to measure a certain physical property. The sensors are of different quality and precision, so they are weighted according to the weighting vector $\boldsymbol{p}=(0.3,0.2,0.2,0.2,0.1)$. Moreover, to prevent a faulty sensor alter the measurement, we take the weighting vector $\boldsymbol{w}=$ $(0,1 / 3,1 / 3,1 / 3,0)$; thus, the maximum and minimum values are not considered.

Given $\boldsymbol{w}=(0,1 / 3,1 / 3,1 / 3,0)$, we have to choose a quantifier interpolating the points $(0,0),(0.2,0),(0.4,1 / 3),(0.6,2 / 3),(0.8,1)$ and $(1,1)$. We consider the quantifier depicted in Figure 1, which is given by

$$
Q(x)= \begin{cases}0 & \text { if } x \leq 0.2 \\ \frac{5}{3} x-\frac{1}{3} & \text { if } 0.2<x<0.8 \\ 1 & \text { if } x \geq 0.8\end{cases}
$$

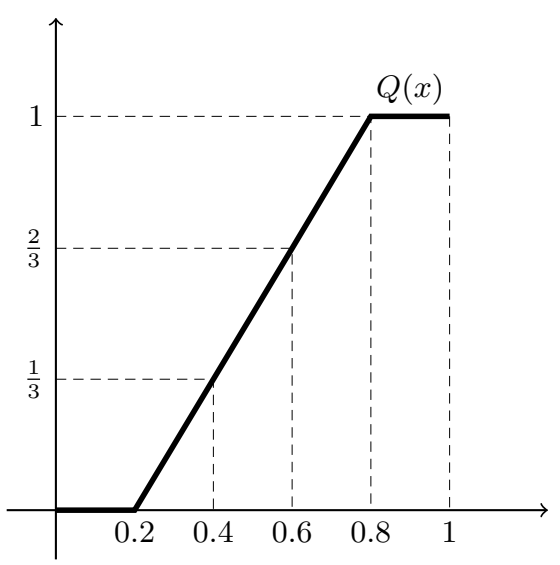

Fig. 1. Quantifier associated to the weighting vector $\boldsymbol{w}=(0,1 / 3,1 / 3,1 / 3,0)$. 
Suppose the values obtained by the sensors are $\boldsymbol{x}=(10,4,5,6,3)$. If $\sigma$ is a permutation ordering these values in a decrease way, then, in this case, $\boldsymbol{p}_{\sigma}=$ $\boldsymbol{p}=(0.3,0.2,0.2,0.2,0.1)$. The weighting vector $\boldsymbol{\mu}$ is

$$
\begin{array}{ll}
\mu_{1}=Q(0.3)-Q(0)=1 / 6, & \mu_{2}=Q(0.5)-Q(0.3)=1 / 3 \\
\mu_{3}=Q(0.7)-Q(0.5)=1 / 3, & \mu_{4}=Q(0.9)-Q(0.7)=1 / 6 \\
\mu_{5}=Q(1)-Q(0.8)=0, &
\end{array}
$$

and $W_{\boldsymbol{p}}^{\boldsymbol{w}}(10,4,5,6,3)=10 / 6+2+5 / 3+4 / 6=6$.

However, our intention is not to consider the maximum and minimum values and only take into account the values 4,5 and 6 ; which have been provided by sensors with the same weight. Therefore, it seems logical to make the average of these values, in which case we would get 5 as final value.

Example 2. Consider again the situation of the previous example and suppose now that $\boldsymbol{p}=(0.4,0.2,0.2,0.1,0.1)$ and $\boldsymbol{x}=(10,3,5,6,7)$. If $\sigma$ is a permutation ordering these values from the largest to the smallest element, then $\boldsymbol{p}_{\sigma}=(0.4,0.1,0.1,0.2,0.2)$. The weighting vector $\boldsymbol{\mu}$ is

$$
\begin{array}{ll}
\mu_{1}=Q(0.4)-Q(0)=1 / 3, & \mu_{2}=Q(0.5)-Q(0.4)=1 / 6, \\
\mu_{3}=Q(0.6)-Q(0.5)=1 / 6, & \mu_{4}=Q(0.8)-Q(0.6)=1 / 3, \\
\mu_{5}=Q(1)-Q(0.8)=0, &
\end{array}
$$

and $W_{\boldsymbol{p}}^{\boldsymbol{w}}(10,3,5,6,7)=10 / 3+7 / 6+1+5 / 3=43 / 6$.

As in the previous example, we do not want to consider the maximum and minimum values and to aggregate the remaining ones, in this case the values 5 , 6 , and 7 . However, the WOWA operator returns a value greater than the three aggregate values because it weights the maximum (10 in this case) with $1 / 3$. On the other hand, there are other interesting properties that the WOWA operator does not satisfy:

1. The value returned by the WOWA operator does not always lie between the values returned by the weighted mean and the OWA operator:

$$
\begin{aligned}
F^{\boldsymbol{w}}(10,3,5,6,7) & =7 / 3+2+5 / 3=6, \\
F_{\boldsymbol{p}}(10,3,5,6,7) & =4+0.6+1+0.6+0.7=6.9,
\end{aligned}
$$

but $W_{\boldsymbol{p}}^{\boldsymbol{w}}(10,3,5,6,7)=43 / 6$.

2. The value returned by the WOWA operator does not always coincide with the values of the weighted mean and the OWA operator when both are equal:

$$
\begin{aligned}
F^{\boldsymbol{w}}(8,2.5,5,6,7) & =7 / 3+2+5 / 3=6, \\
F_{\boldsymbol{p}}(8,2.5,5,6,7) & =3.2+0.5+1+0.6+0.7=6,
\end{aligned}
$$

but $W_{\boldsymbol{p}}^{\boldsymbol{w}}(8,2.5,5,6,7)=8 / 3+7 / 6+1+5 / 3=6.5$. 


\section{Choosing functions to maintain the relationship among the weights}

As we have seen in the previous section, the results provided by WOWA operators may be questionable. If we consider again Example 2, we want to aggregate the values 5,6 , and 7 , which are the values given by the sensors with weights $0.2,0.1$, and 0.1 , respectively. One possibility is to weight these values by means of the weighting vector $(0.5,0.25,0.25)$. In this way, it is possible to maintain the relationship among the initial weights. The returned value in this case is $23 / 4$. by

According to the above remarks, we look for a function $F_{p}^{w}: \mathbb{R}^{n} \longrightarrow \mathbb{R}$ given

$$
F_{\boldsymbol{p}}^{\boldsymbol{w}}\left(x_{1}, \ldots, x_{n}\right)=\sum_{i=1}^{n} \rho_{i} x_{\sigma(i)},
$$

where $\sigma$ is a permutation of $\{1, \ldots, n\}$ such that $x_{\sigma(1)} \geq \cdots \geq x_{\sigma(n)}$ and the weight $\rho_{i}$ is defined as

$$
\rho_{i}=\frac{f\left(w_{i}, p_{\sigma(i)}\right)}{\sum_{j=1}^{n} f\left(w_{j}, p_{\sigma(j)}\right)},
$$

where $f:[0,1]^{2} \longrightarrow[0,1]$. In this way the weights $\rho_{i}$ depend on the weights $w_{i}$ and $p_{\sigma(i)}$.

In order to maintaining the relationship among the weights of a vector ( $\boldsymbol{p}$ or $\boldsymbol{w})$ when the non-zero components of the other vector are equal, it is necessary that $f$ satisfies the following condition:

$$
f(t x, y)=f(x, t y)=t f(x, y),
$$

for all $x, y \in[0,1]$ and $t \in[0, \infty)$ such that $t x, t y \in[0,1]$. In the next proposition we characterize the functions that satisfy this condition.

Proposition 1. Let $f:[0,1]^{2} \longrightarrow[0,1]$ be a function such that $f(t x, y)=$ $f(x, t y)=t f(x, y)$ for all $x, y \in[0,1]$ and $t \in[0, \infty)$ such that $t x, t y \in[0,1]$. Then $f(x, y)=c x y$, where $c \in[0,1]$.

Proof. Given $x, y \in[0,1], f(x, y)=f(x \cdot 1, y \cdot 1)=x f(1, y \cdot 1)=x y f(1,1)$.

$$
\begin{gathered}
\text { If } f(x, y)=c x y \text {, with } c \in[0,1] \text {, then } \rho_{i}=\frac{w_{i} p_{\sigma(i)}}{\sum_{j=1}^{n} w_{j} p_{\sigma(j)}} \text {; that is, } \\
\qquad F_{\boldsymbol{p}}^{\boldsymbol{w}}\left(x_{1}, \ldots, x_{n}\right)=\frac{\sum_{i=1}^{n} w_{i} p_{\sigma(i)} x_{\sigma(i)}}{\sum_{j=1}^{n} w_{j} p_{\sigma(j)}} .
\end{gathered}
$$


It is worth noting that this function has been used by Engemann et al. [1] in a framework of decision making under risk and uncertainty (in this case, $\boldsymbol{p}$ is the vector of probabilities of the states of nature).

In order to ensure that $F_{\boldsymbol{p}}^{\boldsymbol{w}}$ is well defined, we need that $w_{j} p_{\sigma(j)}$ be non-zero for some $j \in\{1, \ldots, n\}$. This requirement is guaranteed by any of the following conditions:

1. The number of non-zero weights in each vector $\boldsymbol{p}$ and $\boldsymbol{w}$ is greater than $n / 2$.

2. All the components of $\boldsymbol{p}$ are non-zero.

In addition to this, $F_{\boldsymbol{p}}^{\boldsymbol{w}}$ has another problem in your definition: sometimes, the vector $\boldsymbol{p}_{\sigma}$ is not unique and $F_{\boldsymbol{p}}^{\boldsymbol{w}}$ may return different values according to the vector $\boldsymbol{p}_{\sigma}$ used. This fact is illustrated in the following example.

Example 3. Consider $\boldsymbol{p}=(0.5,0.2,0.3), \boldsymbol{w}=(0.3,0.4,0.3)$, and $\boldsymbol{x}=(7,5,7)$. When $\boldsymbol{x}$ is ordered from greatest to least, then we have the vector $(7,7,5)$. In this vector, the first component can be associated to the weight 0.5 or 0.3 . In the first case, the weighting vector $\boldsymbol{\rho}$ is $(5 / 11,4 / 11,2 / 11)$ and

$$
F_{\boldsymbol{p}}^{\boldsymbol{w}}(7,5,7)=\frac{35}{11}+\frac{28}{11}+\frac{10}{11}=\frac{73}{11} .
$$

In the second case, the weighting vector $\boldsymbol{\rho}$ is $(9 / 35,4 / 7,6 / 35)$ and

$$
F_{\boldsymbol{p}}^{\boldsymbol{w}}(7,5,7)=\frac{9}{5}+4+\frac{6}{7}=\frac{233}{35} .
$$

A similar problem arises in the IOWA operators, introduced by Yager and Filev [10]. The solution proposed by these authors, applied to our framework, is to replace the weights associated to equal values by the average of them. In the previous example we replace the weights $p_{1}=0.5$ and $p_{3}=0.3$ by 0.4 . In this case the weighting vector $\boldsymbol{\rho}$ is $(6 / 17,8 / 17,3 / 17)$ and

$$
F_{\boldsymbol{p}}^{\boldsymbol{w}}(7,5,7)=\frac{42}{17}+\frac{56}{17}+\frac{15}{17}=\frac{113}{17}
$$

With regard to the properties satisfied by $F_{\boldsymbol{p}}^{\boldsymbol{w}}$, it is easy to check that $F_{\boldsymbol{p}}^{\boldsymbol{w}}$ is idempotent, compensative, homogeneous of degree 1, and that satisfies $F_{\boldsymbol{p}}^{\boldsymbol{\eta}}=F_{\boldsymbol{p}}$ (Engemann et al. [1]) and $F_{\boldsymbol{\eta}}^{\boldsymbol{w}}=F^{\boldsymbol{w}}$.

Nevertheless, as noted by Liu [3], $F_{\boldsymbol{p}}^{\boldsymbol{w}}$ is not monotonic. Moreover, similar to WOWA operators, it does not satisfy other interesting properties as we show in the next example.

Example 4. Consider again the weighting vectors $\boldsymbol{p}=(0.4,0.2,0.2,0.1,0.1)$ and $\boldsymbol{w}=(0,1 / 3,1 / 3,1 / 3,0)$. Then, we have:

1. The value returned by $F_{\boldsymbol{p}}^{\boldsymbol{w}}$ does not always lie between the values returned by the weighted mean and the OWA operator:

$F^{\boldsymbol{w}}(10,3,5,6,7)=6, \quad F_{\boldsymbol{p}}(10,3,5,6,7)=6.9$, but $F_{\boldsymbol{p}}^{\boldsymbol{w}}(10,3,5,6,7)=23 / 4$. 
2. The value returned by $F_{\boldsymbol{p}}^{\boldsymbol{w}}$ does not always coincide with the value returned by the weighted mean and the OWA operator when both values are the same:

$F^{\boldsymbol{w}}(8,2.5,5,6,7)=6, \quad F_{\boldsymbol{p}}(8,2.5,5,6,7)=6$, but $F_{\boldsymbol{p}}^{\boldsymbol{w}}(8,2.5,5,6,7)=23 / 4$

\section{Concluding remarks}

In this paper we have analyzed WOWA operators and we have shown that, in some applications, these operators do not always provide the expected result. Due to the questionable behavior of these operators, we have imposed a condition to maintain the relationship among the weights and we have characterized the functions that satisfy this condition. However, the obtained functions are not monotonic. So, we can conclude that none of the analyzed functions is fully convincing.

Acknowledgments. This work is partially supported by the Junta de Castilla y León (Consejería de Educación y Cultura, Project GR99), the Spanish Ministry of Science and Innovation (Project ECO2009-07332), and ERDF.

\section{References}

1. Engemann, K.J., Filev, D.P., Yager, R.R.: Modelling decision making using immediate probabilities. International Journal of General Systems 24, 281-294 (1996)

2. Fullér, R.: On obtaining OWA operator weights: a short survey of recent developments. In: Fifth IEEE International Conference on Computational Cybernetics (ICCC2007), pp. 241-244, Gammarth, Tunisia (2007)

3. Liu, X.: Preference solutions of probability decision making with RIM quantifiers. International Journal of Intelligent Systems 20, 1253-1271 (2005)

4. Torra, V.: The weighted OWA operator. International Journal of Intelligent Systems 12, 153-166 (1997)

5. Torra, V., Godo, L.: Averaging continuous distributions with the WOWA operator. In: Second European Workshop on Fuzzy Decision Analysis and Neural Networks for Management, Planning and Optimization (EFDAN'97), pp. 10-19, Dortmund, Germany (1997)

6. Torra, V., Narukawa, Y.: Modeling Decisions: Information Fusion and Aggregation Operators. Springer, Berlin (2007)

7. Xu, Z.S.: An overview of methods for determining OWA weights. International Journal of Intelligent Systems 20, 843-865 (2005)

8. Yager, R.R.: On ordered weighted averaging operators in multicriteria decision making. IEEE Transactions on Systems, Man and Cybernetics 18, 183-190 (1988)

9. Yager, R.R.: Families of OWA operators, Fuzzy Sets and Systems 59, 125-148 (1993)

10. Yager, R.R., Filev, D.P.: Induced ordered weighted averaging operators. IEEE Transactions on Systems, Man, and Cybernetics 29, 141-150 (1999) 